\title{
UNA ESPERIENZA DA SCUOLA SULLA DIATERKANEITÁ.
}

Nota del Dott. SILVIO LUSSANA.

Un mezzo molto semplice ed elegante per mostrare ad un numeroso uditorio la trasparenza o l'opacità dei corpi per le radiazioni calorifiche è il seguente, fondato sopra il cangiamento di colore che alcune sostanze soffrono per variazioni di temperatura.

Si incomincia dal preparare dello joduro doppio di argento e mercurio in polvere minutissima. Alla temperatura ordinaria è di un bel colore giallo canarino, mentre che diventa d'un vivo rosso porpora alla temperatura di $49^{\circ}$ circa, per riprendere il colore primitivo quando si raffredda. Questo joduro doppio si prepara secondo il Prof. Bellati ed il Dott. Romanese ") che ne studiarono le proprietà termiche, mescolando in quantità proporzionali ai pesi molecolari le due sostanze $\mathrm{HgI}_{\text {g }}$ e 2(AgI); quindi alla miscela si aggiunge dell' alcool che scioglie in parte lo joduro mercurico, e si stempera e si agita la poltiglia con un pestello, aggiungendo di tratto in tratto dell' alcool. La miscela che dapprima ha un colore rosso, ben presto passa al ranciato, e dopo qualche tempo diventa giallo canarino; allora si lascia evaporare l'alcool rimanente, continuando a stemperare la poltiglia.

Preparato così questo joduro doppio lo si distende su un foglio di carta formando uno schermo di colore giallo canarino sensibile alle radiazioni calorifiche. Se pertanto si porta questo schermo al di sotto di una sfera metallica fortemente riscaldata, si vede lo schermo cambiare tinta, ciò che dimostra il riscaldamento che subisce per irradiazione. La stessa cosa succede se si interpone una lastra di ebanite fra la sfera e lo schermo; ma se per es. sulla lastra di ebanite si dispongono delle lastrine di sostanze opache alle radiazioni calorifi-

1) M. Bellati ed R. Romanese. - Proprietà termiche notevoli di alcuni joduri doppi. (Nuovo Cimento (3) 8, 215, 1880). 
che, si vede sullo schermo proiettata nettamente l'ombra di queste lastrine, comparendo tale o. ${ }^{3}$ bra di un colore giallo in un campo rosso. Con questo mezzo si capisce che si può ottenere anche la proiezione dell' ombra di corpi atermani racchiusi fra due lastrine di ebanite o di tutt'altra sostanza diatermana.

Quest' esperienza riesce con tutta facilità e potrà sostituire l'esperienza analoga fatta col banco del Melloni, avendo il vantaggio di mostrare a colpo d' occhio che una stessa sostanza puó essere trasparente per certe radiazioni, mentre è opaca per altre.

Dall' Istituto Fisico dell' Università di Siena,

Maggio 1896.

SUI RAGGI RÖNTGEN.

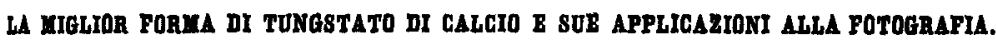
SPLANCNOSCOPIO.

Note del Dott. FERDINANDO GIAZZI (Perugia).

(Sunto del Dott. A. I'ET'ROZZANI).

L'A., dopo di aver riconosciuto che il tungstato di calcio ottenuto pestando le perle fuse al gas ossidrico era meno fluorescente ai raggi Röntgen del baricianuro di platino, dice che se si scalda il tungstato di calcio amorfo e secco in un fornello a Coke alimentato da una corrente di ossigeno o di aria, il tungstato non si liquefä, ma si riduce a strüttura saccaroide, che ridotto in polvere finissima e di nuovo scaldato come prima prende l'aspetto del marmo di Carrara, aspetto che l'A. chiama bisaccaroide. Per preparare la carta al tungstato l'A. ne distende un foglio su di un telaio, lo ricopre di una densa soluzione di gomma arabica, vi staccia sopra della polvere di tungstato e la lascia asciugare; dipoi con carta a smeriglio rende la superficie omogenea. L'A. poi descrive, uno strumento, 